\title{
Implantação do Processo de Medição Aderente ao Modelo MR-MPS-SW com Foco em Estudo de Tempos em Empresas com Times SCRUM
}

Tipo do Artigo: Relato de Experiência

\author{
Mariana Alves Moura ${ }^{12}$, Victor José Aguiar Teixeira de Melo França ${ }^{12}$, Ana \\ Cristina Rouiller ${ }^{1}$ \\ ${ }^{1}$ UFRPE - Universidade Federal Rural de Pernambuco \\ ${ }^{2}$ SWQ - SWQuality Consultoria e Sistemas \\ \{mariana@swquality.com.br, victoreswquality.com.br, \\ ana.rouilleregmail.com\}
}

\begin{abstract}
This paper is an experience report of the application of the methodology for implementation of Measurement and Analysis process in groups of companies, developed by SWQuality under the SWImprovement project (MCT/CNPq) with the support of UFRPE - Universidade Federal Rural de Pernambuco. The measurement process was implemented through the adoption of agile practices based on SCRUM methodology and using Times Study concepts within a program of implantation of Improvement of Software Process based on the level F of the reference model MR-MPS-SW, in a group of companies of evolution maintenance of software.
\end{abstract}

Resumo. Este trabalho relata a experiência da aplicação da metodologia para implantação do processo de Medição e Análise em grupos de empresas, desenvolvida pela SWQuality no âmbito do projeto SWImprovement $(M C T / C N P q)$ com apoio da UFRPE - Universidade Federal Rural de Pernambuco. O processo de Medição foi implantado por meio da adoção de práticas ágeis fundamentadas na metodologia SCRUM e utilizando conceitos do Estudo de Tempos, dentro de um programa de implantação de Melhoria de Processos de Software baseado no nível F do modelo de referência MR-MPSSW, em um grupo de empresas de evolução e manutenção de software.

\section{Introdução}

Diante da grande competitividade do mercado de Tecnologia da Informação (TI), para garantir seu espaço no mercado, as empresas brasileiras estão cada vez mais investindo em implementações de modelos de melhoria de processos de software, um deles é o MPS.BR - Melhoria de Processos de Software Brasileiro. De acordo com a Softex:

"O MPS.BR é um programa mobilizador que foi criado em 2003 pela Softex para melhorar a capacidade de desenvolvimento de software nas empresas brasileiras. A iniciativa foi responsável pelo desenvolvimento do Modelo de Referência para Melhoria do Processo de Software Brasileiro (MR-MPS-SW) que levou em consideração normas e modelos internacionalmente reconhecidos, boas práticas da engenharia de software e as necessidades de negócio da indústria de software nacional [...] a fim de estabelecer um caminho economicamente viável para que organizações, incluindo as pequenas e 
médias empresas, alcancem os benefícios da melhoria de processos e da utilização de boas práticas da engenharia de software em um intervalo de tempo razoável"1.

Alinhado a isso, muitas empresas buscam uma maneira mais leve de gerenciar o trabalho, o que torna bastante prudente a inclusão de práticas ágeis combinadas com estes modelos. Uma metodologia ágil amplamente utilizada por indústrias de software e que vem dando muito certo é o SCRUM (Schwaber, 2011), um processo de desenvolvimento iterativo e incremental para o gerenciamento de projetos e desenvolvimento de software ágil. Já existem estudos que demonstram a eficiência de unir métodos ágeis com a implantação de melhoria de processos de software, realizando algumas adaptações nas técnicas propostas por esses métodos para que eles tornem-se aderentes às práticas solicitadas pelos modelos, como é possível verificar em Catunda (2011), Salgado (2010), Marçal (2009), Jakobsen (2009), Pikkarainen (2006), Salo (2005), entre outros.

Partindo deste princípio, este relato de experiência apresenta uma importante etapa do trabalho de implantação do nível F do MR-MPS-SW combinado com a metodologia ágil SCRUM em um grupo de 7 pequenas empresas, com times compostos de 3 a 9 pessoas, que trabalham com desenvolvimento, evolução e manutenção de software. Esta etapa tratou da aplicação da metodologia para implantação do processo de Medição e Análise em grupos de empresas, desenvolvida pela SWQuality no âmbito do projeto SWImprovement (MCT/CNPq) com apoio da UFRPE - Universidade Federal Rural de Pernambuco. O principal objetivo deste processo foi desenvolver na organização a capacidade de avaliar, de maneira mais objetiva, o desempenho da sua célula produtiva, permitindo que as tomadas de decisão pudessem ser feitas de maneira mais consciente pelos gestores, com base em dados e não apenas em palpites. Este processo é essencial para que as empresas possam se sentir mais seguras ao competir num ambiente caracterizado por tantas mudanças como é o mercado de TI.

No processo de Medição, uma maior visibilidade dos resultados da empresa é viabilizada a partir da definição de indicadores de desempenho que podem responder a diferentes objetivos pré-definidos pela organização. Para este trabalho de implantação, foram usados conceitos do Estudo de Tempos (Barnes, 1977), uma importante área da Administração Científica criada por Frederick Winslow Taylor. Este estudo foi usado como embasamento para a concepção de métricas a partir dos dados gerados pelo processo de desenvolvimento ágil adotado.

\section{O Processo de Medição no Modelo de Referência MR-MPS-SW}

O processo de Medição compõe o nível F do modelo de referência MR-MPS-SW e é um dos mais importantes pois possui interseção com todos os outros processos. Além disso, analisado sob o ponto de vista gerencial e estratégico, ele é de grande relevância para apoiar na tomada de decisões relacionadas ao desenvolvimento dos projetos, aos processos e aos objetivos organizacionais.

Como explicitado no Guia de Implementação Parte 2 do MPS.BR (SOFTEX, 2013), "as medições são criadas de forma organizada a partir dos objetivos organizacionais e necessidades estratégicas de informação da organização". Dessa forma, este processo

\footnotetext{
${ }^{1}$ Disponível em: <http://www.softex.br/mpsbr/mps/mps-br-em-numeros/> Acesso em 02 abril 2015.
} 
deve responder às questões da organização de tal forma a apoiar no julgamento, de maneira mais objetiva, da eficiência e eficácia das equipes e dos processos e da viabilidade dos projetos e negócios.

Os resultados esperados com a implementação do processo de Medição podem ser conferidos na Tabela 1.

Tabela 1 - Resultados esperados do processo de Medição de acordo com o MRMPS-SW

\begin{tabular}{|c|l|}
\hline ID & \multicolumn{1}{|c|}{ Resultado Esperado } \\
\hline MED 1 & $\begin{array}{l}\text { Objetivos de medição são estabelecidos e mantidos a partir dos objetivos de } \\
\text { negócio da organização e das necessidades de informação de processos } \\
\text { técnicos e gerenciais; }\end{array}$ \\
\hline MED 2 & $\begin{array}{l}\text { Um conjunto adequado de medidas, orientado pelos objetivos de medição, é } \\
\text { identificado e definido, priorizado, documentado, revisado e, quando } \\
\text { pertinente, atualizado; }\end{array}$ \\
\hline MED 3 & $\begin{array}{l}\text { Os procedimentos para a coleta e o armazenamento de medidas são } \\
\text { especificados; }\end{array}$ \\
\hline MED 4 & Os procedimentos para a análise das medidas são especificados; \\
\hline MED 5 & Os dados requeridos são coletados e analisados; \\
\hline MED 6 & Os dados e os resultados das análises são armazenados; \\
\hline MED 7 & $\begin{array}{l}\text { Os dados e os resultados das análises são comunicados aos interessados e são } \\
\text { utilizados para apoiar decisões. }\end{array}$ \\
\hline
\end{tabular}

\section{O Uso do Estudo de Tempos Alinhado à Concepção de Métricas nos Times SCRUM}

O Estudo de Tempos é uma maneira de mensurar o trabalho a partir de métodos estatísticos, que pretende avaliar e planejar a mão-de-obra em qualquer sistema produtivo. Este estudo teve origem na Administração Científica, quando o engenheiro Frederick Taylor verificou a necessidade de analisar e padronizar os métodos de trabalho para atingir a eficiência produtiva. Como princípios da filosofia de Taylor, destacam-se: os operários devem realizar durante o dia de trabalho uma produção aceitável; existe um método adequado para todo e qualquer trabalho; é preciso instruir o trabalhador para que possa realizar o trabalho adequadamente; é preciso fixar condições para o trabalho; é necessário fixar um tempo padrão para o trabalho (Chiavenato, 1997). Dentre os principais benefícios do Estudo de Tempos, destacam-se: eliminar operações desnecessárias, determinar a real capacidade produtiva da equipe, facilitar a administração da produção, melhorar as condições ergonômicas de trabalho, projetar novas oportunidades e outros.

Alinhadas a esses princípios trazidos pela Administração e a partir da institucionalização do SCRUM nos times de desenvolvimento, métricas foram concebidas tendo como embasamento o Estudo de Tempos que foi realizado na célula produtiva das empresas a partir da inserção da prática do apontamento de horas das tarefas realizadas pelos colaboradores. Por dividir o trabalho em ciclos curtos (Sprints) e 
de mesma duração, o SCRUM permite a padronização do trabalho e alguns dados, como a capacidade produtiva da equipe dentro de um determinado período, o custo de desenvolvimento em horas e o tempo médio gasto em retrabalho, entre outros puderam ser extraídos. Essas métricas puderam servir de insumos para a criação de indicadores que auxiliassem os gestores em suas decisões.

\section{Implantação do Processo de Medição nas empresas}

A implantação do processo de Medição nas empresas fez parte de um programa de implementação do Nível F do modelo de referência MR-MPS-SW. Este projeto de implantação foi dividido em etapas (vide Tabela 2), quase todas provendo alguma contribuição para a implantação do processo de Medição e foi gerado um conjunto de indicadores para apoiar os gestores das organizações.

\subsection{Etapas do Programa de Implementação do MR-MPS-SW e suas Relações com a Implantação do Processo de Medição}

Um resumo das etapas da Implantação do MR-MPS-SW e seus relacionamentos com o processo de Medição pode ser observado na Tabela 2.

Tabela 2 - Etapas da Implantação de Melhoria e seu relacionamento com o processo de Medição

\begin{tabular}{|l|l|}
\hline \multicolumn{2}{|c|}{ Fase 1-Apresentação do Projeto e Nivelamento } \\
\hline Descrição & $\begin{array}{l}\text { Apresentação do Programa de Melhoria de Processos para as } \\
\text { empresas participantes a fim de esclarecer possíveis dúvidas e } \\
\text { orientá-las na estruturação do ambiente propício para a realização } \\
\text { dos trabalhos. Nesta fase também foi realizado um treinamento } \\
\text { para nivelamento dos colaboradores das empresas envolvidos } \\
\text { diretamente com o projeto de implantação do MR-MPS-SW. }\end{array}$ \\
\hline $\begin{array}{l}\text { Relacionamento } \\
\text { com o Processo } \\
\text { de Medição }\end{array}$ & $\begin{array}{l}\text { O processo de Medição assim como os demais processos do Nível } \\
\text { de nivelamento dos colaboradores. } \\
\text { Resultados Esperados Relacionados: Nenhum. }\end{array}$ \\
\hline \multicolumn{1}{|c|}{ Fase 2 - Diagnóstico e Planejamento } \\
\hline Descrição & $\begin{array}{l}\text { Identificação do nível de aderência dos processos existentes ao } \\
\text { nível F do MR-MPS-SW, entendimento da empresa, identificação } \\
\text { de problemas e expectativas com o programa de melhoria. A partir } \\
\text { das informações coletadas foi realizado um planejamento em alto } \\
\text { nível considerando as metas estabelecidas para o projeto. }\end{array}$ \\
\hline $\begin{array}{l}\text { Relacionamento } \\
\text { com o Processo } \\
\text { de Medição }\end{array}$ & $\begin{array}{l}\text { No diagnóstico foram coletados alguns objetivos estratégicos dos } \\
\text { gestores e algumas necessidades de informação que posteriormente } \\
\text { serviram de insumo para a definição dos indicadores. } \\
\text { Resultados Esperados Relacionados: MED 1. }\end{array}$ \\
\hline \multicolumn{1}{|c|}{ Fase 3 - Implantação do SCRUM } \\
\hline Descrição & $\begin{array}{l}\text { Os processos atuais do setor de desenvolvimento foram } \\
\text { reformulados conforme o programa de melhoria da qualidade }\end{array}$ \\
\hline
\end{tabular}




\begin{tabular}{|c|c|}
\hline & $\begin{array}{l}\text { utilizando como referência a metodologia ágil SCRUM e foi feito } \\
\text { um acompanhamento da introdução e adaptação da metodologia no } \\
\text { ambiente das empresas. }\end{array}$ \\
\hline $\begin{array}{l}\text { Relacionamento } \\
\text { com o Processo } \\
\text { de Medição }\end{array}$ & $\begin{array}{l}\text { Com a gestão de times seguindo a metodologia SCRUM, foi } \\
\text { iniciado o Estudo de Tempos a partir da inserção da técnica de } \\
\text { apontamento de horas trabalhadas e a estimativa das tarefas de } \\
\text { desenvolvimento em pontos. Por dividir a gestão em ciclos curtos e } \\
\text { de tempo fixo, o SCRUM auxiliou na padronização do trabalho e } \\
\text { permitiu que métricas pudessem ser extraídas por meio dos dados } \\
\text { gerados. } \\
\text { Resultados Esperados Relacionados: MED } 2 \text {. }\end{array}$ \\
\hline \multicolumn{2}{|r|}{ Fase 4 - Abordagem do Primeiro Grupo de Processos } \\
\hline Descrição & $\begin{array}{l}\text { Nesta fase foi dado ênfase aos processos de Gerência de Requisitos } \\
\text { e Gerência de Projetos. Foram definidos processos para atender as } \\
\text { exigências do modelo e estes foram executados em projetos piloto. } \\
\text { Estes processos foram implantados e monitorados e adaptados caso } \\
\text { necessário. }\end{array}$ \\
\hline $\begin{array}{l}\text { Relacionamento } \\
\text { com o Processo } \\
\text { de Medição }\end{array}$ & $\begin{array}{l}\text { Na implantação da Gerência de Projetos a gestão do } \\
\text { desenvolvimento passou a ser realizada seguindo um Plano de } \\
\text { Projeto. Neste plano alguns objetivos e parâmetros de } \\
\text { acompanhamento são definidos pelo Gerente de Projetos. Estes } \\
\text { objetivos e parâmetros serviram posteriormente como insumo para } \\
\text { a definição dos indicadores. } \\
\text { Resultados Esperados Relacionados: MED } 1\end{array}$ \\
\hline \multicolumn{2}{|r|}{ Fase 5 - Abordagem do Segundo Grupo de Processos } \\
\hline Descrição & $\begin{array}{l}\text { Nesta fase são trabalhados os processos de Gerência de } \\
\text { Configuração, Garantia da Qualidade e Medição. Foram definidos } \\
\text { processos para atender as exigências do modelo e estes foram } \\
\text { executados em projetos piloto. Estes processos foram implantados } \\
\text { e monitorados e adaptados caso necessário. }\end{array}$ \\
\hline $\begin{array}{l}\text { Relacionamento } \\
\text { com o Processo } \\
\text { de Medição }\end{array}$ & $\begin{array}{l}\text { O processo de Medição foi trabalhado com maior ênfase, } \\
\text { buscando-se estratégias para satisfazer a todos os resultados } \\
\text { esperados do modelo de referência. A partir dos objetivos e } \\
\text { necessidades de medição já identificados e das métricas criadas, foi } \\
\text { criado um Guia de Medição onde estas informações foram } \\
\text { registradas e relacionadas a indicadores. Neste documento também } \\
\text { foram definidos os procedimentos de coleta e armazenamento e os } \\
\text { procedimentos de análise dessas informações. Em seguida foi } \\
\text { definida a estratégia para a execução do processo, que compreende } \\
\text { a coleta e análise dos dados, seu armazenamento e a comunicação } \\
\text { dos resultados. O processo foi executado, monitorado e adaptado, } \\
\text { caso necessário, em projetos piloto nas organizações. } \\
\text { Resultados Esperados Relacionados: MED 1, MED 2, MED 3, } \\
\text { MED 4, MED 5, MED 6, MED 7. }\end{array}$ \\
\hline
\end{tabular}




\section{Fase 6 - Maturação dos Processos}

\begin{tabular}{|l|l|}
\hline Descrição & $\begin{array}{l}\text { Nesta fase foi realizada a institucionalização dos processos em } \\
\text { todos os projetos das empresas. Durante a institucionalização, a } \\
\text { utilização dos processos foi monitorada e possíveis desvios e } \\
\text { melhorias foram identificadas e implementadas. }\end{array}$ \\
\hline $\begin{array}{l}\text { Relacionamento } \\
\text { com o Processo } \\
\text { de Medição }\end{array}$ & $\begin{array}{l}\text { Monitoramento da utilização do processo de Medição e avaliação } \\
\text { da sua aderência aos resultados esperados do MR-MPS-SW. Todos } \\
\text { os resultados esperados foram revisados e, caso necessário, } \\
\text { aprimorados nesta fase. } \\
\text { Resultados Esperados Relacionados: MED 1, MED 2, MED 3, } \\
\text { MED 4, MED 5, MED 6, MED 7. }\end{array}$ \\
\hline \multicolumn{1}{|c|}{ Fase 7- Encerramento } \\
\hline $\begin{array}{l}\text { Descrição } \\
\text { Relacionamento } \\
\text { com o Processo } \\
\text { de Medição }\end{array}$ & $\begin{array}{l}\text { Nesta fase foi realizada uma avaliação simulada indicando se as } \\
\text { empresas estão aptas a submeter-se a uma avaliação oficial ou se } \\
\text { ainda serão necessários alguns ajustes. Para concluir, um workshop } \\
\text { foi realizado e as empresas apresentaram umas para as outras os } \\
\text { seus resultados. } \\
\text { Resultados esperados foram verificados a fim de serem } \\
\text { MED 4, MED 5, MED 6, MED 7. }\end{array}$ \\
\hline
\end{tabular}

\subsection{Resultados da Implantação do Processo de Medição}

A implantação de Medição nas empresas foi realizada a partir do Estudo de Tempos provido pelo apontamento de horas pelos colaboradores, que foi inserido na Fase 3 da implantação do MR-MPS-SW, como pode ser conferido na Tabela 2. A partir dos dados obtidos com este estudo e pelas estimativas geradas em cada ciclo do SCRUM, alguns indicadores puderam ser concebidos na Fase 5 (vide Tabela 2) e utilizados para auxiliar os gestores nas tomadas de decisão. Alguns desses indicadores podem ser conferidos abaixo:

- Horas Trabalhadas X Horas Disponíveis: Este indicador verifica o percentual de horas trabalhadas em relação à carga-horária da equipe de desenvolvimento do projeto.

- Índice de Retrabalho: Este indicador verifica o percentual de horas gastas pela equipe em retrabalho, em relação ao total de horas gastas. A meta da empresa é cada vez mais reduzir o valor obtido nesse indicador pois isso indica que a equipe está trabalhando em melhorias dos sistemas e em novas funcionalidades em vez de corrigir erros.

- Horas Trabalhadas Por Projeto: Algumas empresas do grupo possuem vários projetos em andamento e cada um deles tem suas prioridades. Este indicador verifica se estas prioridades estão sendo respeitadas. $\mathrm{O}$ indicador consiste em medir o percentual de horas despendidas em cada projeto. 
- Custo do Ponto: Em todas as empresas foi inserida a técnica de estimar o trabalho pontuando os requisitos de acordo com a dificuldade de implementação. No final de cada ciclo do SCRUM é coletado o total de pontos trabalhados e as horas gastas para desenvolver esses pontos para calcular o indicador Custo do Ponto, que consiste em dar uma ideia de quanto tempo é gasto para que seja desenvolvido um ponto.

- Horas Gastas Por Tipo de Atividade: Este indicador consiste no percentual de horas gastas em cada tipo de atividade do processo de desenvolvimento das empresas. Nas ferramentas de gestão das empresas foram realizadas configurações para apoiar na triagem das atividades, sejam de desenvolvimento, teste, planejamento, monitoramento, qualidade, gerência de configuração, reuniões, entre outras.

Ao final do processo de implantação, todas as empresas do grupo foram avaliadas no modelo MR-MPS-SW e todas obtiveram a certificação.

\section{Conclusões}

Este trabalho relatou a experiência da implantação do processo de Medição aderente ao modelo MR-MPS-SW, aplicando a metodologia para implantação do processo de Medição e Análise em grupos de empresas, desenvolvida pela SWQuality e usando o Estudo de Tempos em empresas que utilizam SCRUM como metodologia de desenvolvimento de software.

O Estudo de Tempos foi realizado por meio da técnica do apontamento de horas e combinado com os dados providos a partir dos ciclos do SCRUM para a formulação de indicadores capazes de prover informações valiosas aos gestores das empresas, para que eles possam tomar suas decisões de maneira mais consciente e objetiva.

Ao final do processo de implantação, todas as empresas do grupo foram avaliadas no modelo MR-MPS-SW e todas obtiveram a certificação, comprovando a aderência da estratégia de implantação utilizada.

\section{Agradecimentos}

Os autores agradecem ao CNPq, à SWQuality e às empresas participantes do Programa de Melhoria.

\section{Referências}

Barnes, R. M. (1977). Estudo de movimentos e de tempos: projeto e medida do trabalho. Editora Edgard Blu cher.

Catunda, E., Nascimento, C., Cerdeiral, C., Santos, G., Nunes, E., Schots, N. C. L., \& Rocha, A. R. (2011). Implementação do Nível F do MR-MPS com Práticas Ágeis do Scrum em uma Fábrica de Software. X Simpósio Brasileiro de Qualidade de Software (SBQS'11), Curitiba-Brasil.

Chiavenato, I. (1997). Teoria Geral da Administração. 5. ed. São Paulo: Makron Books.

Jakobsen, C. R., \& Sutherland, J. (2009). Scrum and CMMI going from good to great. In Agile Conference, 2009. AGILE'09. (pp. 333-337). IEEE. 
Marçal, A. S. C. (2009). SCRUMMI: Um processo de gestão ágil baseado no SCRUM e aderente ao CMMI. Universidade de Fortaleza.

Pikkarainen, Minna, and Annukka Mantyniemi. "An approach for using CMMI in agile software development assessments: experiences from three case studies." (2006).

Salgado, A., Melcop, T., Acchar, J., Rego, P. A., Ferreira, A. I. F., Katsurayama, A. E., \& Zanetti, D. (2010). Aplicação de um Processo Ágil para Implantação de Processos de Software baseado em Scrum na Chemtech. IX Simpósio Brasileiro de Qualidade de Software (SBQS).

Salo, O., \& Abrahamsson, P. (2005, November). Integrating agile software development and software process improvement: a longitudinal case study. In Empirical Software Engineering, 2005. 2005 International Symposium on (pp. 10-pp). IEEE.

Santana, C. A., Timóteo, A. L., \& Vasconcelos, A. M. (2006). "Mapeamento do modelo de melhoria do processo de software brasileiro (mps. br) para empresas que utilizam extreme programming (xp) como metodologia de desenvolvimento." V Simpósio Brasileiro de Qualidade de Software [SBQS], 5.

Schwaber, K., \& Sutherland, J. (2011). The scrum guide. Scrum Alliance.

SOFTEX, MPS.BR. Disponível em: <http://www.softex.br/mpsbr/mps/mps-br-emnumeros/> Acesso em 02 abril 2015.

SOFTEX - Associação para Promoção da Excelência do Software Brasileiro (2013), "Melhoria do Processo de Software Brasileiro - Guia de Implementação - Parte 2: Fundamentação para Implementação do Nível F do MR-MPS-SW:2012”. 